\title{
Sputtering of Vacuum Deposited Metal Films by Oxygen Ion Bombardment
}

\author{
Yoshiaki Okajima* and Yukiyoshi Aizawa*
}

(Received November 8, 1977)

\begin{abstract}
The sputtering rates of chromium, copper, germanium and gold films, which were prepared by a vacuum deposition method, were measured after oxygen ion bombardment. It was found that sputtering rates are proportional to the current density of primary oxygen ions. This relation can be used to determine the depth of a sputtered surface when beam parameters change, and allows to obtain the compositional depth profile of multi-layer metal films, prepared by vacuum deposition.
\end{abstract}

\section{Introduction}

An ion microanalyzer is often used to determine the compositional depth profiles of multi-layer metal films deposited on metal and semiconductor substrates. In the compositional depth profiles reported up to now, elemental composition has usually been shown as a function of bombardment time. It would, however, be more desirable to give it as a function of depth. If the rate of sputtering of an element and the sputtering time are known, then the depth of a sputtered surface can be determined. Almen and Bruce ${ }^{1)}$ measured the sputtering rates of various elements and summarized the rates as a function of atomic number. In other experiments, it was found that sputtering rates are affected by the species, energy and incident angle of the ion beam used for bombardment ${ }^{1-4)}$. And Rol $^{\text {s) }}$, Thompson ${ }^{6)}$, Sigmund ${ }^{2)}$ and others have investigated sputtering phenomena theoretically. However, the specimens used in these studies were single and poly-crystal block specimens.

In this paper, the sputtering rates of film specimens, that is, metal films deposited on silicon and silicon dioxide substrate, are investigated. It is assumed that the sputtering rate of a metal film, prepared by vacuum deposition, and of the block metal are different, because the number of crystal lattice faults in the film is larger than the number in the block. A method is proposed to determine the depth of a sputtered surface, enen when beam parameters change. It is also shown that the

*Hitachi Research Lab., Hitachi Ltd., 4026, Kuji-machi, Hitachi-shi, Ibaraki 319-12. 
sputtering rate and depth resolution of metal films and of blocks are different.

\section{Experimental}

\subsection{Preparation of metal films.}

Chromium, copper, germanium or gold were deposited on a $<111>$ surface of an n-type silicon wafer in a conventional vacuum system. The silicon substrate was chemically etched with a mixed solution of hydrofluoric and nitric acids. Evaporation took place at a pressure of less than $0.7 \mathrm{mPa}$. The substrate was heated up $300^{\circ} \mathrm{C}$ by a quartz lamp and kept at that temperature during deposition. High purity chromium powder, granulated copper, germanium powder or gold wire was evaporated from a tungsten boat. The deposition rates of the chromium, copper, germanium and gold were $34,92,80$ and $63 \mathrm{~nm} / \mathrm{min}$, respectively.

In the preparation of a multi-layer film, chromium, copper and gold were de posited, in that order, on a silicon dioxide substrate under the same conditions as described above.

The thickness of single layer films of chromium, copper, germanium and gold were $0.22,1.02,2.10$ and $0.98 \mu \mathrm{m}$, respectively. And the total thickness of the multi-layer film was about $1 \mu \mathrm{m}$.

\subsection{Experimental procedure.}

An Hitachi type IMA-2 Ion Microanalyzer was used in this study. Typical sput tering conditions are listed in Table. 1. A hollow cathode duoplasmatron was used as the oxygen ion source. The ion were accelerated toward the sample to have the kinetic energy of $10 \mathrm{keV}$. The beam was
Table 1. Working conditions for sputtering experiment.

\begin{tabular}{l|l}
\hline $\begin{array}{l}\text { Ionic Species } \\
\text { Accelerating voltage for } \\
\text { primary ions }\end{array}$ & $10 \mathrm{keV}$ \\
$\begin{array}{l}\text { Current density of primary ions } \\
\text { Beam diameter }\end{array}$ & $0.4-3.7 \mu \mathrm{A} / \mathrm{mm}^{2}$ \\
Pressure in sample chamber & $27 \mu \mathrm{Pa}$ \\
$\begin{array}{l}\text { Accelerating voltage for } \\
\text { secondary ions }\end{array}$ & $3 \mathrm{keV}$ \\
\hline
\end{tabular}

focused by two condenser lenses so that the beam diameter was in the range of about 0.5 to $1.0 \mathrm{~mm}$. The primary ion current was in the range of 0.2 to $0.5 \mu \mathrm{A}$. The sample chamber was evacuated to approximately $27 \mu \mathrm{Pa}$.

The depth of the sputtered surface after oxygen ion bombardment was measured with a 138-000 type Sloan Dektak. A diamond stylus, $5 \mu \mathrm{m}$ in diameter, scanned the sputtered surface of the metal film. A difference of about $10 \mathrm{~nm}$ between the unsputtered and sputtered surfaces of a specimen can be detected with this method.

The sputtering rate $(\mathrm{nm} / \mathrm{min})$ of metal film was determined from the relation between the sputtered depth and sputtering time.

\section{Results}

\subsection{Roughness of sputtered surface.}

Figure 1 shows the texture of the surfaces of copper specimens sputtered by oxygen ion bombardment. Accelerating voltage, current and the diameter of the primary ion beam were $10 \mathrm{kV}, 0.5 \mu \mathrm{A}$ and $0.8 \mathrm{~mm}$, respectively. There is pronounced surface unevenness in case of the copper block. On the other hand, the surface of 


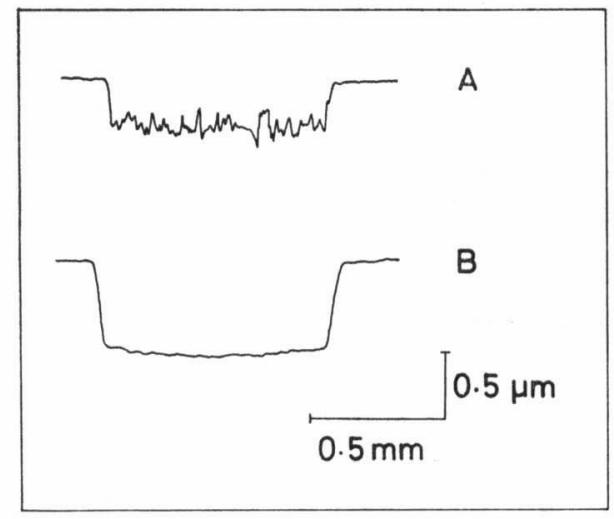

Fig. 1 Roughness of sputtered surfaces of copper specimens. (A) block and (B) film.

Primary ions were oxygen ions and bombardment time was $60 \mathrm{~min}$.

Accelerating voltage, current and diameter of primary ion beam were $10 \mathrm{keV}, 0.5 \mu \mathrm{A}$ and $0.8 \mathrm{~mm}$, respectively.

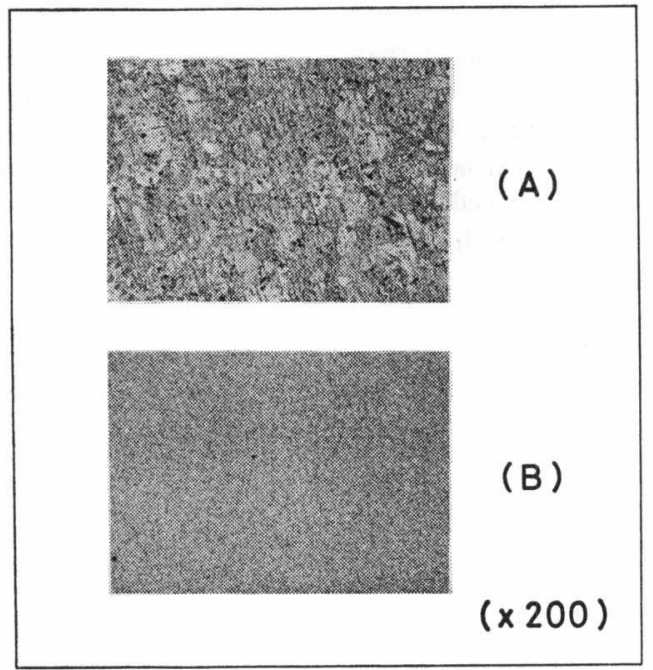

Fig. 2 Photographs of sputtered surface.

Specimens were the same as in Fig. 1. (A) Block and (B) film. Sputtering conditions were the same as in Fig. 1.

the copper film is very smooth. Grain boundaries can be observed on the sputter ed surface of the block, but not on the sputterred surface of the film, as shown by the photographs in Fig. 2. Apparently, the surface of the film is smooth and the grain structure of the film is fine compared with the case of the block.

The sputtering depth of the film is greater than that of the block, as shown in Fig. 1. Figure 3 shows a comparison of

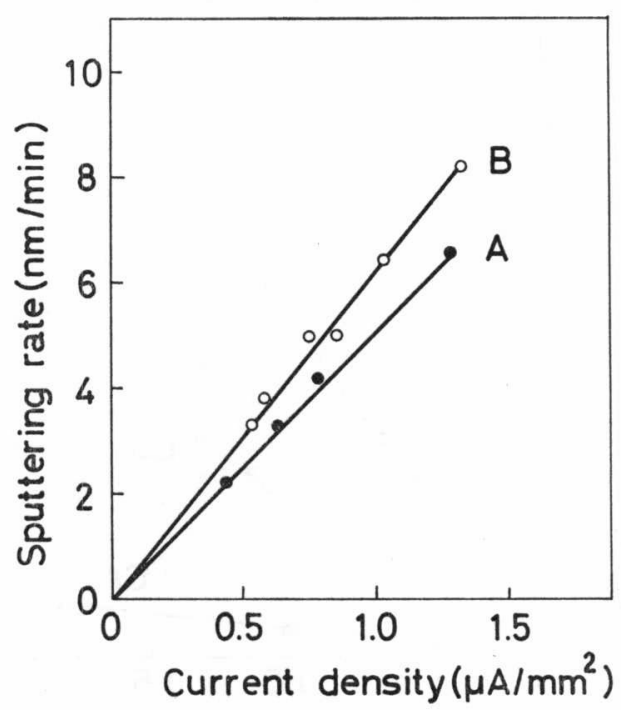

Fig. 3 Comparison of sputtering rate of

(A) block and of (B) film, using copper specimens. Current and diameter of primary oxygen ion beam were 0.2 to 0.5 $\mu \mathrm{A}$ and 0.4 to $0.8 \mathrm{~mm}$, respectively.

the sputtering rate for film and for block. When the current density of the primary oxygen ions is the same for a film and a block, the sputtering rate of the film is larger than that of the block.

\subsection{Sputtering rates of various metal films.}

The sputtering rates of chromium, copper, germanium and gold films, prepared by vacuum deposition, were measured with a scanning stylus after oxygen ion bombard- 
ment. These results were summarized in the relation between sputtering rate and the current density of the oxygen ions. All of the films measured in this report show a linear relationship between sputtering rate and current density, as shown in Fig. 4. When the current or diameter

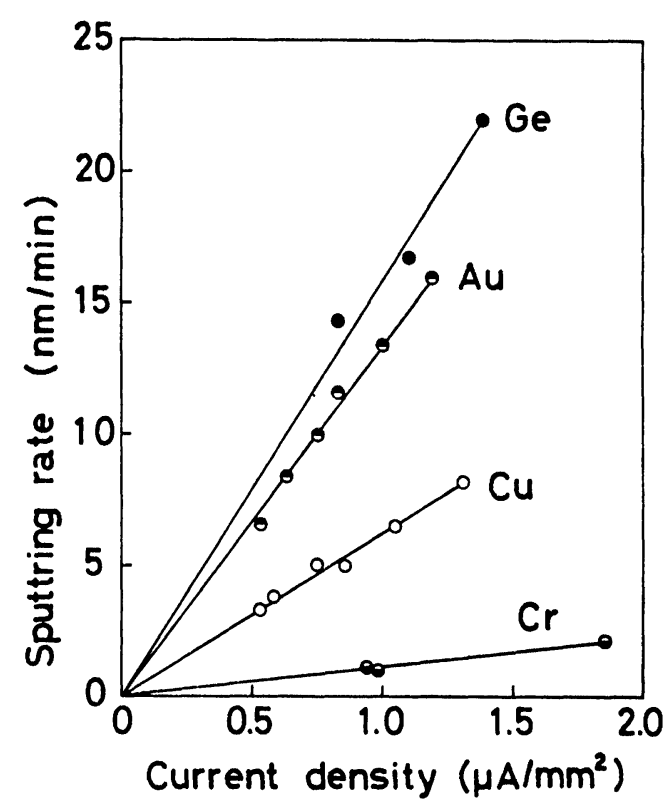

Fig. 4. Relationship between sputtering rates of films and current density of primary oxygen ions. Current and diameter of primary ion beam were 0.2 to $0.5 \mu \mathrm{A}$ and 0.4 to $1.1 \mathrm{~mm}$, respectively.

of the primary ion beam vary, the sputtering rate of a film can be determined with the above relation.

The slope of the curve of the relation is different from one element to another. It is thought that these differences depend upon the binding energies of the elements ${ }^{1}$.

\subsection{Analysis of multi-layer film specimen.}

The above results were applied to the determination of the compositional depth profile of a multi-layer film of chromium, copper and gold which were deposited in that order on a silicon dioxide substrate. Figure 5 shows the obtained profile. The

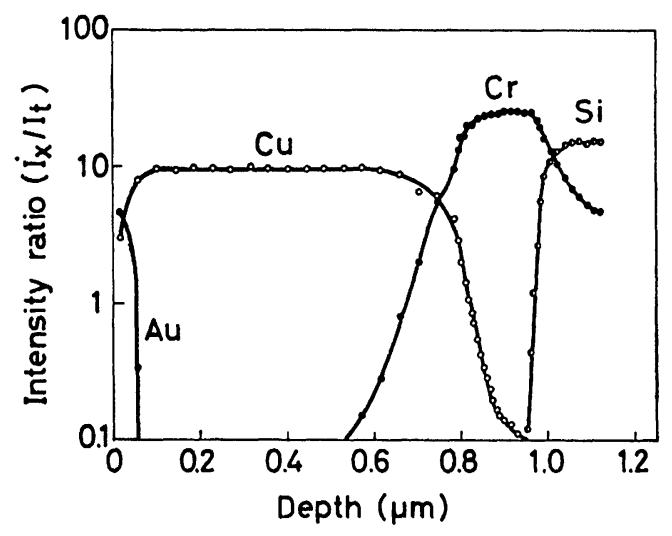

Fig. 5 Compositional depth profile of multi-layer film of chromium, copper and gold, deposited in that order on a silicon dioxide substrate. Current density of primary oxygen ions was $2.1 \mu \mathrm{A} / \mathrm{mm}^{2}$ (current: 0.4 $\mu \mathrm{A}$, beam diameter: $0.5 \mathrm{~mm}$ ). $\mathrm{I}_{x}$ is the secondary ion intensity of each element and $I_{t}$ is the total ion intensity.

thickness of each layer was calculated using the date shown in Fig. 4 and the bombardment time. The time required for sputtering each layer was taken as the time that the intensity fell to one-half of its maximum value.

The sputtering rate in a layer boundary where two kinds of metals are mixed could not be determined from the sputtering rates of the two pure metals, because sputtering rate and the concentration of the metals in an alloy are nonlinearly related. Therefore, the point where the intensity was one-half of its maximum value was plotted for each of the two metals. 
Sputtering of vacuum deposited metal films by oxygen ion bombardment

The center point between these two points was taken as the boundary of two metals.

The total thickness obtained for the chromium, copper and gold film in scanning stylus measurements was $1.0 \mu \mathrm{m}$. The thickness shown in Fig. 5 agrees with the value obtained in the scanning stylus measurements.

\section{References}

1) O. Almen and G. Bruce, Nucl. Inst. Methods, 11, 257 (1961).
2) P. Sigmund, Phys. Sev., 184, 383 (1969).

3) R. J. MacDonald, Advan. Phys., 19, 457 (1970).

4) J. M. Fluit, R. K. Rol and J. Kistemaker, J. Appl. Phys. 34, 690 (1963).

5) P. K. Rol, J.M. Fluit and J. Kistemaker, Physica, 26, 1009 (1960).

6) M. W. Thompson, Phil. Mag., 18, 377 (1968).

\section{Keywords}

Ion bombardment Sputtering phenomena Ion microanalyser Vacuum deposited film In-depth analysis 